\title{
Immunoadsorption therapy reduces oxidative stress in patients with dilated cardiomyopathy
}

\author{
Hiroki Kasai $^{* *}$, Yuichiro Kashima ${ }^{1^{*}}$, Atsushi Izawa ${ }^{1}$, Takeshi Tomita ${ }^{1}$, Yusuke Miyashita ${ }^{1}$, \\ Jun Koyama ${ }^{1}$, Masafumi Takahashi ${ }^{2}$, Taku Yoshio ${ }^{3}$, Yoshikazu Yazaki ${ }^{4}$, \\ Makoto Higuchi $^{5}$, Uichi Ikeda ${ }^{1 \#}$ \\ ${ }^{1}$ Department of Cardiovascular Medicine, Shinshu University School of Medicine, Nagano, Japan \\ ${ }^{2}$ Division of Bio-Imaging, Jichi Medical University, Tochigi, Japan \\ ${ }^{3}$ Department of Rheumatology and Clinical Immunology, Jichi Medical University, Tochigi, Japan \\ ${ }^{4}$ Division of Cardiology, Matsumoto Medical Center, Nagano, Japan \\ ${ }^{5}$ Department of Nephrology, Shinshu University School of Medicine, Nagano, Japan \\ Email: ${ }^{*}$ uikeda@shinshu-u.ac.jp
}

Received 10 August 2012; revised 15 September 2012; accepted 23 September 2012

\section{ABSTRACT}

Several literatures have reported that the elimination of autoantibodies by immunoadsorption (IA) therapy induced short- and long-term improvements in cardiac function of patients with dilated cardiomyopathy (DCM). We assessed whether reduction in oxidative stress was related to the mechanism underlying left ventricular functional benefit from IA. We studied 7 patients with DCM (New York Heart Association (NYHA) functional class III/IV). Autoantibodies were removed with IA by passing patients' plasma over tryptophan columns. The level of anti- $\beta 1$-adrenoreceptor (AR) autoantibodies was measured by enzymelinked immunosorbent assay. The level of diacronreactive oxygen metabolite (d-ROM) was determined as a marker of oxidative stress. During IA, the anti$\beta 1-A R$ autoantibodies titers of all the patients decreased significantly from $27.8 \pm 5.0$ to $18.7 \pm 5.5$ $\mathrm{U} / \mathrm{ml}(\mathbf{p}<0.01)$. IA induced the following hemodynamic improvements: cardiac index increased from $1.71 \pm 0.40$ to $1.97 \pm 0.41 \mathrm{l} / \mathrm{min} / \mathrm{m}^{2}$ and left ventricular ejection fraction (LVEF) increased from $22.8 \pm 6.1$ to $29.1 \pm 9.1 \%(p<0.05)$. d-ROM level decreased significantly from $392.7 \pm 17.0$ to $314.1 \pm 22.0$ Carratelli units $(p<0.05)$, and was negatively correlated with LVEF before and after IA therapy $(r=-0.601, p<$ $0.05)$. The anti- $\beta 1-A R$ autoantibodies, LVEF and $d-$ ROM returned to the baseline levels at 12 months after IA. In conclusion, oxidative stress reduction may be responsible for the beneficial effect of IA therapy in patients with DCM.

Keywords: Autoantibody; Immunoglobulin;

${ }^{*}$ H.K. and Y.K. contributed equally to this work.

${ }^{\#}$ Corresponding author.
Plasmapheresis

\section{INTRODUCTION}

Dilated cardiomyopathy (DCM) is a chronic myocardial disease characterized by progressive ventricular enlargement and myocardial contractile malfunction [1]. DCM is not a rare cause of congestive heart failure and the leading reason for heart transplantation world wide [2]. Although the fundamental mechanisms responsible for the development and progression of left ventricular failure have not yet been completely elucidated, a variety of experimental studies suggest that alterations in the immune system might be involved in the pathogenesis of DCM [3]. Several autoantibodies directed against cardiac cellular proteins, including G-protein-linked receptors, contractile proteins, and mitochondrial proteins, have been identified in patients with DCM. Among these, the pathogenic role of autoantibodies against $\beta 1$-adrenoreceptors (ARs), belonging to immunoglobulin (Ig) G3, has been well investtigated in experimental models $[4,5]$ and human DCM [6-8]. In addition, several literatures reported that the elimination of anti- $\beta 1-\mathrm{AR}$ autoantibodies with immunoadsorption (IA) therapy induced short- and long-term improvements in the left ventricular ejection fraction (LVEF), cardiac output, and New York Heart Association (NYHA) functional class in patients with DCM, who were refractory to conventional medical therapy.

IA is a new therapeutic approach for patients with DCM, and the mechanism underlying left ventricular functional benefit from IA is not well understood. IgG adsorption removes not only anti- $\beta 1-\mathrm{AR}$ autoantibodies but also all other potentially pathogenic autoantibodies in this class of IgGs affect the heart. Mobini et al. [9] has reported that the effect of hemodynamic improvement during IA was 
similar among patients positive and negative for anti$\beta 1$-AR autoantibodies, suggesting that the beneficial effects of IA may not be directly associated with the selective elimination of anti- $\beta 1$-AR autoantibodies.

Recent experimental and clinical studies have suggested that the generation of reactive oxygen species (ROS) such as superoxide anions $\left(\mathrm{O}^{2-}\right)$ and hydroxy radicals $(\cdot \mathrm{OH})$ are increased in chronic heart failure [10]. An imbalance between ROS and antioxidant reserve, referred to as oxidative stress, alters the structure and function of membrane phospholipids, proteins, and mitochondrial DNA, resulting in enhanced myocardial remodeling and cardiac functional failure [11]. In patients with DCM, left ventricular dysfunction is thought to be partly caused by oxidative stress [12]. Previously, Schimke et al. [13] reported that IA therapy with anti-oxidant supplementation such as vitamins, minerals, and trace elements resulted in simultaneous improvement in cardiac performance and reduction in oxidative stress one year after the therapy. However, thus far there have been no reports on the early and direct effect of IA therapy on oxidative stress. Hence, in the present study, we investigated the effect of IA therapy on oxidative stress and cardiac and clinical performance of patients with DCM by assessing changes of these parameters before and after IA therapy.

\section{METHODS}

\subsection{Study Population}

A total of 7 patients with DCM, who were admitted and had undergone IA at our hospital from December 2007 to December 2008 were enrolled in this study. All the patients had severe left ventricular dysfunction (NYHA functional class III or IV; LVEF $<30 \%$, cardiac index $<$ $2.21 / \mathrm{min} / \mathrm{m}^{2}$, as established by two-dimensional echocardiography, and Swan-Ganz thermodilution catheterization, respectively). Patients were excluded if they had a history of active infectious disease, cancer, chronic alcoholism, or heart failure due to other known origins such as coronary artery disease, primary valvular heart disease, and acute myocarditis. Disease duration for all the patients was longer than 2 years, which suggests chronic myocardial processes. Written consent was obtained from each patient before enrolment in this study, and the protocol was approved by the Ethics Committees at Shinshu University School of Medicine.

\subsection{Immunoadsorption Therapy}

The extracorporeal IA system consisted of conventional plasmapheresis and immunoapheresis systems. We used a plasma filter (Plasmaflo $\mathrm{OP}^{\circledR}$; Asahi Kase Kuraray Medical, Japan) for plasmapheresis and a tryptophan column (Immusorba $\mathrm{TR}^{\circledR}$; Asahi Kase Kuraray Medical) that contained a matrix of cross-linked polyvinyl alcohol gel beads to which the hydrophobic amino acid tryptophan could be immobilized for immunoglobulin extraction from the plasma [14]. We separated $2000 \mathrm{ml}$ of plasma using the IA column, and performed IA therapy twice a week; total 3 sessions for first 3 patients and 5 sessions for subsequent 4 patients without immunoglobulin or fibrinogen substitution.

\subsection{Hemodynamics}

For hemodynamics measurement, two-dimensional cardiac echocardiography was performed at baseline and immediately after the final IA session, and 12 months after IA therapy. LVEF was assessed according to the Simpson rule, and the left ventricular internal diameter in diastole (LVDd) was measured. To evaluate the hemodynamics, including cardiac index and pulmonary capillary wedge pressure (PCWP), we performed right heart catheterization using a Swan-Gnaz thermodilution catheter at just before the first session and immediately after the final session.

\subsection{Sampling and Measurements}

For measuring of the level of anti- $\beta 1$-AR autoantibodies, we performed an enzyme-linked immunosorbent assay (ELISA). We used synthesized peptides corresponding to the sequence of the second extracellular loop of the human $\beta 1$-AR (Peptide Institute Inc., Minoh, Japan). We incubated 96-well microtiter plates (Sumitomo Bakelite, Tokyo, Japan) for $2 \mathrm{~h}$ at $37^{\circ} \mathrm{C}$ with $100 \mu \mathrm{l} /$ well of WSC (1-ethyl-3-(3-dimethylaminopropyl) carbodiimide hydrochloride; Dojindo, Kumamoto, Japan) at a concentration of $10 \mathrm{mg} / \mathrm{ml}$ in phosphate-buffered saline (PBS), $\mathrm{pH}=5.8$. After 3 washes with $200 \mu \mathrm{l} /$ well of PBS, we coated the wells with $100 \mu \mathrm{l} /$ well of the synthetic peptide-bovine serum albumin (BSA) conjugates at a concentration of 5 $\mu \mathrm{g} / \mathrm{ml}$ of the synthetic peptide in PBS and incubated the plates at $37^{\circ} \mathrm{C}$ overnight. The wells were blocked at $4^{\circ} \mathrm{C}$ overnight with Block Ace (Dainippon Pharmaceutical, Osaka, Japan) that was diluted 1:4 with distilled water. Serum samples were diluted 1:500 in PBS containing 1\% BSA and added to the synthetic peptide conjugate-coated wells, and the plates were left at room temperature for 2 $\mathrm{h}$. The rest of the process was performed as previously described [15]. The titers for the anti- $\beta 1$-AR autoantibodies were determined on the basis of a standard curve of optical density using high-titer positive serum as previously reported and expressed as arbitrary units $/ \mathrm{ml}$.

We determined the levels of diacron-reactive oxygen metabolite (d-ROM) and biological antioxidant potential (BAP), as markers of oxidative stress, in the venous serum samples collected just before and after IA therapy, 
and 12 months after IA therapy. The d-ROM level represents the total amount of peroxidized metabolites [16], and BAP reflects serum antioxidant capacity [17]. Values for d-ROM and BAP were ascertained by the Free Radical Elective Evaluator (Diacron International, Grosseto, Italy) using commercial assay kits (Diacron srl, Grosseto, Italy). The d-ROM level is proportional to the serum hydroperoxide concentration. Hydroperoxides are the peroxidation products of proteins, peptides, amino acids, lipids, and fatty acids. Measurement of the d-ROM level is based on the ability of transition metals to catalyze, in the presence of peroxides, the formation of free radicals, which are trapped by an alchilamine. The alchilamine reacts to form a colored radical that can be detected at $505 \mathrm{~nm}$. The results were expressed in conventional units called U.CARR (Carratelli units), and 1 U.CARR corresponds to $0.8 \mathrm{mg} / 1 \mathrm{H}_{2} \mathrm{O}_{2}$. Normal levels of d-ROM range between 250 and 300 U.CARR. BAP measurement is based on the ability of a colored solution, containing a source of ferric $\left(\mathrm{Fe}^{3+}\right)$ ions bound to a chromogenic substrate (thiocyanate derivative), to decolor when $\mathrm{Fe}^{3+}$ ions are reduced to ferrous ions $\left(\mathrm{Fe}^{2+}\right)$ by the reducing activity of blood samples. Chromatic change was read using a photometer at $505 \mathrm{~nm}$. Preliminary data from healthy individuals indicate that a normal BAP value is $>2200$ $\mu \mathrm{mol} / 1$.

The concentration of tumor necrosis factor- $\alpha$ (TNF- $\alpha$ ) was determined using a commercially available Becton Dickinson (BD) cytometric bead array human Th1/Th2 cytokine kit II (BD Biosciences, San Jose, CA, USA) according to the manufacturer's instructions. Briefly, serum or cytokine standards were added to a mixture of Capture Beads and Human Th1/Th2-PE Detection Reagent. The mixture was incubated for $3 \mathrm{~h}$ at room temperature and protected from exposure to light. Subsequently, the beads were washed to remove unbound detector Capture Beads and Human Th1/Th2-PE Detection Reagent. Flow cytometry was performed using FACSCaliber (BD Biosciences, San Jose, CA, USA). Data were acquired and analyzed using CellQuest software (BD Biosciences, San Jose, CA, USA) and BD Cytometric Beads Array software (BD Biosciences, San Jose, CA, USA).

\subsection{Statistical Analysis}

Statistical analysis was performed using Prism Ver.4 (GraphPad Software, Inc., CA, USA). The changes in IgG3, anti- $\beta 1$-AR autoantibodies, d-ROM, BAP, TNF- $\alpha$ and BNP between before and after IA were analyzed by paired $t$-test. Pearson's correlation coefficient test was used to analyze the difference between the level of $d-$ ROM and LVEF or TNF- $\alpha$. Results were expressed as mean \pm SEM. $p$ values $<0.05$ were considered statisti- cally significant.

\section{RESULTS}

Table 1 summarizes the baseline characteristics of the 7 patients (mean age: $62.4 \pm 13.6$ years; range: $35-74$ years) included in this study. A male patient with NYHA class IV had been mechanically supported by intra-aortic balloon counterpulsation (IABP) for a month, because he developed pre-cardiogenic shock with oliguria. At baseline, all the patients had been administered maximal tolerated dosages of long-term oral medications on a stable basis for at least more than 6 months, including $\beta$-blocker, angiotensin II receptor blocker, digoxin, or diuretic, and had not received antioxidant supplementation such as vitamins, and minerals. Stable medication and dosage across the patients did not differ during the study. All the patients tolerated IA therapy, and neither clinical nor laboratory data revealed any signs of complication, including infectious disease, hemorrhage, and renal failure.

After the sessions of IA therapy, the titers of IgG3 and anti- $\beta 1-\mathrm{AR}$ autoantibodies of all the patients decreased from $63.5 \pm 15.0$ to $14.6 \pm 2.9 \mathrm{mg} / \mathrm{dl}(\mathrm{p}<0.01)$ and from $27.8 \pm 5.0$ to $18.7 \pm 5.5 \mathrm{U} / \mathrm{ml}(\mathrm{p}<0.01)$, respectively (Figures 1A and B). However, these parameters returned to the baseline levels at 12 months $(55.4 \pm 16.0 \mathrm{mg} / \mathrm{dl}$ and $26.4 \pm 9.5 \mathrm{U} / \mathrm{ml}$, respectively). Cardiac death was observed by 12 months after IA therapy in 2 patients.

IA therapy improved the following hemodynamic characteristics (Table 2): Cardiac index increased from $1.71 \pm 0.40$ to $1.97 \pm 0.411 / \mathrm{min} / \mathrm{m}^{2}(\mathrm{p}<0.05)$, and LVEF increased from $22.8 \% \pm 6.1 \%$ to $29.1 \% \pm 9.1 \%(p<0.05)$ after IA. However, at 12 months, the changes of LVEF became not significant $(34.4 \% \pm 12.8 \%)$ (Figure 2). Indicators such as PCWP, BNP, LVDd did not change significantly after IA therapy.

An examination of the NYHA classification revealed improvement in 3 and 2 out of the 7 patients at 1 month and 12 months after IA. The classification of the patient with class IV before IA did not change, but IABP could be withdrawn immediately after IA.

Table1. Baseline characteristics of 7 patients.

\begin{tabular}{ll}
\hline Age (years) & $62.4 \pm 13.6$ \\
Gender (male/female) & $5 / 2$ \\
NYHA classification III/IV & $6 / 1$ \\
Medications (\%) & \\
ARB & $7(100)$ \\
$\beta$-blocker & $6(86)$ \\
Digoxin & $3(43)$ \\
Diuretics & $5(71)$ \\
\hline
\end{tabular}

NYHA = New York Heart Association; ARB = Angiotensin II receptor blocker. 


\section{(A) IgG3 subclass}

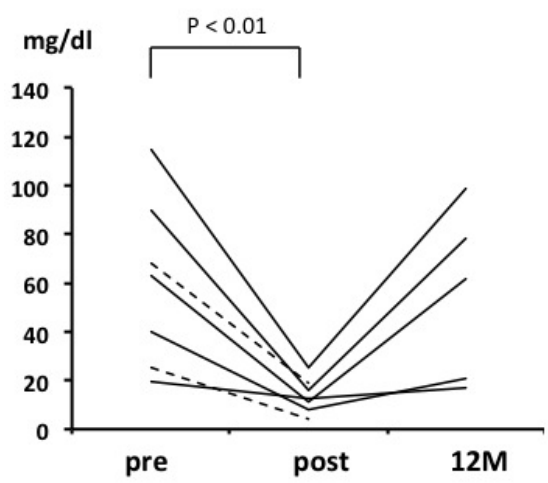

(B) Anti- $\beta 1$ AR Ab

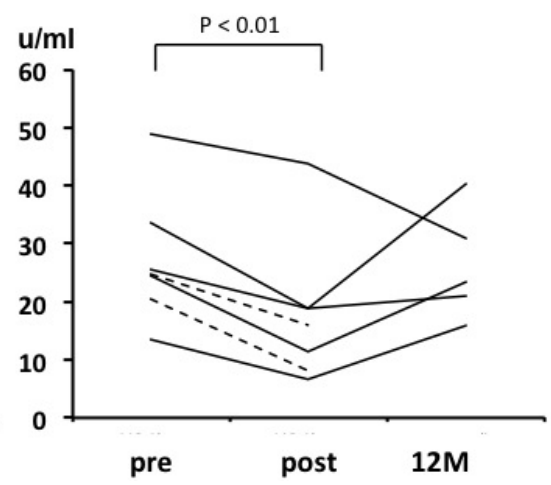

Figure 1. Individual data points of all the patients for $\operatorname{IgG} 3(\mathrm{~A})$ and anti- $\beta 1-\mathrm{AR}$ autoantibodies (B) at pre-, post-, and 12 months after immunoadsorption. The dotted lines represent 2 patients died by 12 months.

Table 2. Effects of IA on cardiac parameters.

\begin{tabular}{llll}
\hline & Pre-IA & Post-IA & p value \\
\hline PCWP $(\mathrm{mmHg})$ & $15.5 \pm 10.6$ & $14.3 \pm 10.3$ & $\mathrm{~ns}$ \\
Cardiac index $\left(\mathrm{I} / \mathrm{min} / \mathrm{m}^{2}\right)$ & $1.71 \pm 0.40$ & $1.97 \pm 0.41$ & $<0.05$ \\
BNP (pg/ml) & $1183 \pm 1063$ & $1045 \pm 967$ & $\mathrm{~ns}$ \\
LVEF (\%) & $22.8 \pm 6.1$ & $29.1 \pm 9.1$ & $<0.05$ \\
LVDd (mm) & $64.1 \pm 9.3$ & $64.1 \pm 9.6$ & $\mathrm{~ns}$ \\
\hline
\end{tabular}

$\mathrm{IA}=$ Immunoadsorption; PCWP = Pulmonary capillary wedge pressure; BNP = Brain natriuretic peptide; LVEF = Left ventricular ejection fraction; LVDd = Left ventricular internal diameter in diastole.
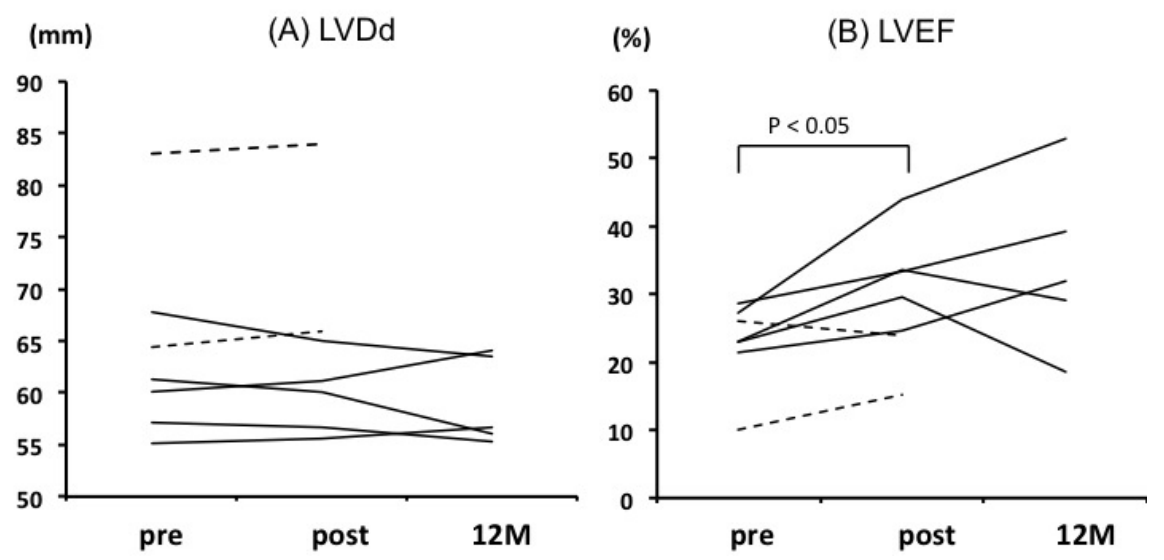

Figure 2. Individual data points of all the patients for LVDd (A) and LVEF (B) at pre-, post-, and 12 months after immunoadsorption. LVDd: left ventricular internal diameter in diastole; LVEF: left ventricular ejection fraction. The dotted lines represent 2 patients died by 12 months.

The level of d-ROM was high at baseline and decreased significantly from $392.7 \pm 17.0$ to $314.1 \pm 22.0$ U.CARR after IA $(\mathrm{p}<0.05)$, while it returned to the baseline level at 12 months $(405.8 \pm 102.9$ U.CARR $)$ (Figure 3). The BAP level, which was normal value at baseline, did not change significantly immediately after IA (from $2318.0 \pm 187.3$ to $2413.0 \pm 148.8 \mu \mathrm{mol} / \mathrm{l}, \mathrm{p}=$
0.56 ), but was significantly increased at 12 months $(3350.6 \pm 348.1 \mu \mathrm{mol} / \mathrm{l}, \mathrm{p}<0.05)$.

As shown in Figure 4, significant inverse correlations were found between d-ROM and LVEF $(r=-0.601, p<$ 0.05 ) before and after IA. On the other hand, the level of anti- $\beta 1$-AR autoantibodies was not associated with LVEF $(\mathrm{r}=-0.063, \mathrm{p}=0.83)$. 
(A) dROM

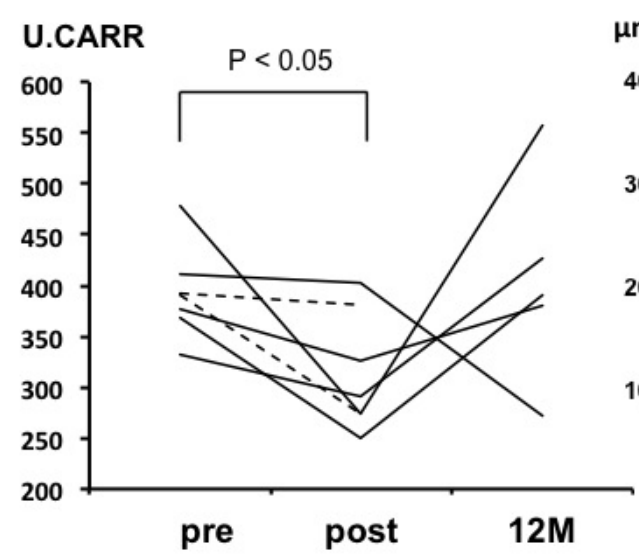

(B) BAP

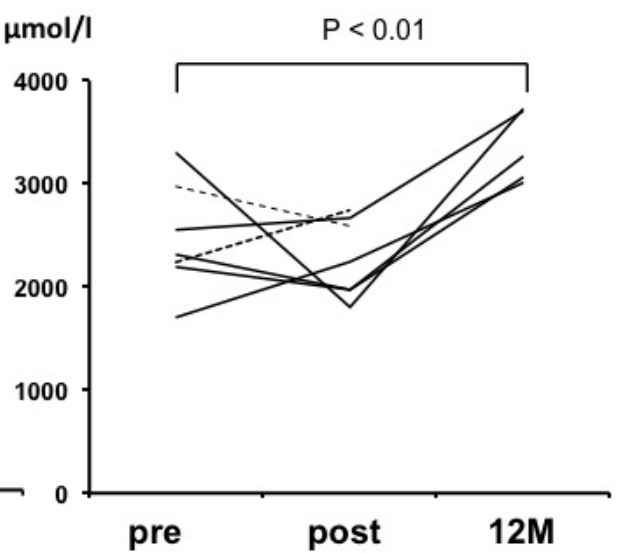

Figure 3. Individual data points of all the patients for d-ROM (A) and BAP (B) at pre-, post-, and 12 months after immunoadsorption. BAP: Biological antioxidant potential; d-ROM: Diacron-reactive oxygen metabolite. The dotted lines represent 2 patients died by 12 months.

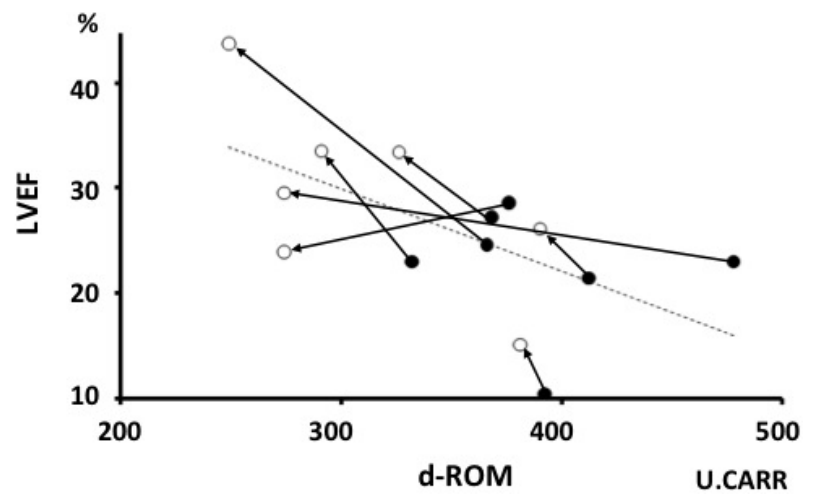

Figure 4. Relation between LVEF and d-ROM. $\bullet=$ Pre-, $\mathrm{O}=$ Post-immunoadsorption. d-ROM: Diacron-reactive oxygen metabolite; LVEF: Left ventricular ejection fraction.

The level of serum TNF- $\alpha$ also significantly decreased from $1.41 \pm 0.81$ to $0.17 \pm 0.45 \mathrm{pg} / \mathrm{ml}(\mathrm{p}<0.01)$ after IA (Figure 5A), and the reduction of TNF- $\alpha$ was significantly correlated to the reduction of the d-ROM level $(\mathrm{r}=$ $0.855, \mathrm{p}<0.05)$ (Figure 5B).

\section{DISCUSSION}

In the present study, we showed that the short-term improvement in cardiac performance after IA therapy in patients with DCM might be related to the reduction in oxidative stress. The mechanisms underlying the action of IA therapy have not yet been elucidated. Although several studies, as well as our present study, have demonstrated that IA decreases the level of circulating anti- $\beta 1$-AR autoantibodies $[6,13,18,19]$, although a cause-and-effect relationship has not yet been established. Mobini et al. [9] has reported that hemodynamic improvement after IA was similar across patients irrespective of whether they were positive or negative for anti- $\beta 1-\mathrm{AR}$ autoantibodies, suggesting that the beneficial effect of IA is not directly associated with the selective elimination of anti- $\beta 1-A R$ autoantibodies. The Immunosorba $\mathrm{TR}^{\circledR}$ column has nonselective physical features, but causes marked reduction in plasma levels of $\mathrm{IgG} 3$ [14]. In our protocol, plasma IgG and IgG3 levels dropped at an average of $37 \%$ and $58 \%$ per IA procedure, respectively. IgG adsorption might remove not only anti$\beta 1$-AR-autoantibodies but also other potentially pathogenic autoantibodies in this class of IgG that can affect the heart.

In the present study, the reduction in d-ROM was significantly correlated with LVEF before and after IA, while the reduction in anti- $\beta 1$-AR autoantibodies was not associated with LVEF, suggesting that an improvement in oxidative status, in addition to the elimination of anti$\beta 1$-AR-autoantibodies, might be responsible for the improvement of cardiac function by IA therapy. Cumulative evidences suggest that the production of oxygen radicals increases in failing hearts [9]. An increased oxidative stress contributes to heart failure, because of enhanced myocyte growth, apoptosis, and fibroblast proliferation [20]. Mitochondrial electron transport is an enzymatic source of oxygen radical generation [21]. Chronic increase in oxygen radical production in the mitochondria can lead to a catastrophic cycle of mitochondrial DNA damage as well as functional decline, further oxygen radical generation, and cellular injury [19]. ROS induce myocyte hypertrophy, apoptosis, and interstitial fibrosis by activating matrix metalloproteinases $[22,23]$. These cellular events play an important role in the development and progression of maladaptive myocardial remodeling and failure.

TNF- $\alpha$ stimulates oxidative stress in the failing hearts of patients with DCM [11,12,24]. Oda et al. [25] investigated the in vitro cytokine adsorptive properties of 
(A)

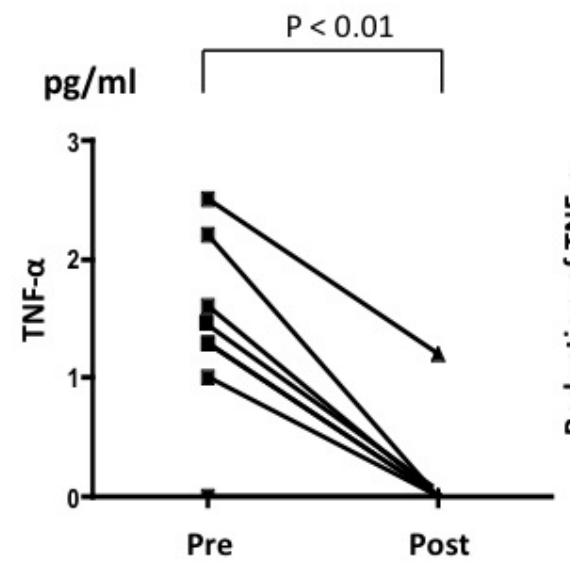

(B)

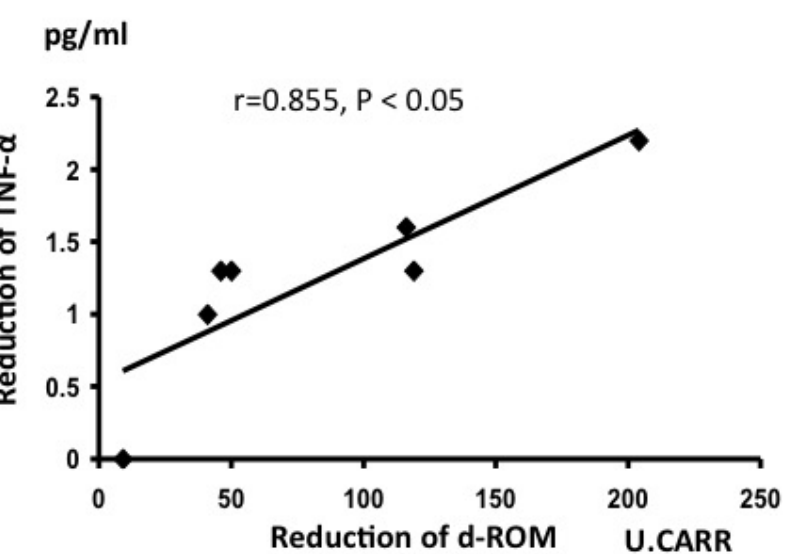

Figure 5. Individual data points of all the patients for serum TNF- $\alpha$ at pre- and post-immunoadsorption (A). Relation between the reduction of TNF- $\alpha$ and d-ROM (B). d-ROM: Diacron-reactive oxygen metabolite.

various plasma adsorbents and found that Immusorba $\mathrm{TR}^{\circledR}$ almost completely removed TNF- $\alpha$. Indeed, in our study, the level of TNF- $\alpha$ was markedly decreased after the sessions of IA therapy, and the reduction in TNF- $\alpha$ level was found to be correlated to the reduction in dROM level, suggesting that the adsorption of TNF- $\alpha$ by IA may be related to the reduction in oxidative stress. In addition, IA therapy reduced the level of d-ROM immediately after IA, while it did not increase the level of BAP; this suggests that IA therapy improves oxidative stress status by reducing the generation of peroxidants rather than by the increasing antioxidant defense.

Improvement in LVEF and reduction of anti- $\beta 1$-AR autoantibodies and d-ROM were observed only in a shortterm period in our study, and a long-term follow-up did not demonstrate the improvement, although the BAP level was significantly increased at 12 months. On the other hands, Muller et al. [26] and Dorffel et al. [27] reported that IA therapy improved the cardiac performance in 1- and 3-year follow-up with persistent decrease in circulating anti- $\beta 1-A R$ autoantibodies. The difference in the results might be due to the protocol of IA; immunoadsorption was performed on 5 consecutive days with immunoglobulin substitution in their protocol, while we performed gentle IA therapy; twice a week, total 3 or 5 sessions without immunoglobulin substitution. Further studies are required to elucidate the long-term effects of IA therapy.

\section{STUDY LIMITATIONS}

We speculated that oxidative stress may be partly responsible for left ventricular dysfunction in patients with DCM and beneficial effects of IA therapy, it is unclear whether it is the causative or resultant factor of heart failure. In addition, reduction in the levels of serum in- dicators of oxidative stress might not directly reflect the reduction in oxidative stress in the heart itself, which is a more favorable indicator of cardiac function.

The number of patients in this study was small and the observation period was only 12 months after IA. Further studies are required to understand the mechanism underlying left ventricular functional benefit from IA in DCM patients.

In conclusion, the reduction in oxidative stress could be one of the mechanisms underlying early beneficial effect of IA therapy in patients with DCM.

\section{ACKNOWLEDGEMENTS}

This study was supported by research grants from the Grants-Aid for Scientific Research (\# 23790842) and the Ministry of Health, Labor and Welfare in Japan.

\section{REFERENCES}

[1] Richardson, P., McKenna, W., Bristow, M., Maisch, B., Mautner, B., O'Connell, J., Olsen, E., Thiene, G., Goodwin, J., Gyarfas, I., Martin, I. and Nordet, P. (1996) Report of the 1995 World Health Organization/International Society and Federation of Cardiology Task Force on the definition and classification of cardiomyopathies. Circulation, 93, 841-842. doi:10.1161/01.CIR.93.5.841

[2] Taylor, D.O., Edwards, L.B., Boucek, M.M., Trulock, E.P., Waltz, D.A., Keck, B.M. and Hertz, M.I. (2006) Registry of the International Society for Heart and Lung Transplantation: Twenty-third official adult heart transplantation report. Journal of Heart and Lung Transplantation, 25, 869-879. doi:10.1016/j.healun.2006.05.002

[3] Maisch, B., Ristić, A.D., Hufnagel, G. and Pankuweit, S. (2002) Pathophysiology of viral myocarditis: The role of humoral immune response. Cardiovascular Pathology, 11, 112-122. doi:10.1016/S1054-8807(01)00113-2 
[4] Iwata, M., Yoshikawa, T., Baba, A., Anzai, T., Nakamura, I., Wainai, Y., Takahashi, T. and Ogawa, S. (2001) Autoimmunity against the second extracellular loop of beta1adrenergic receptors induces beta-adrenergic receptor desensitization and myocardial hypertrophy in vivo. Circulation Research, 88, 578-586.

doi:10.1161/01.RES.88.6.578

[5] Jahns, R., Boivin, V., Hein, L., Triebel, S., Angermann, C.E., Ertl, G. and Lohse, M.J. (2004) Direct evidence for a beta1-adrenergic receptor-directed autoimmune attack as a cause of idiopathic dilated cardiomyopathy. Journal of Clinical Investigation, 113, 1419-1429.

[6] Müller, J., Wallukat, G., Weng, Y.G., Dandel, M., Spiegelsberger, S., Semrau, S., Brandes, K., Theodoridis, V., Loebe, M., Meyer, R. and Hetzer, R. (1997) Weaning from mechanical cardiac support in patients with idiopathic dilated cardiomyopathy. Circulation, 96, 542-549. doi:10.1161/01.CIR.96.2.542

[7] Jahns, R., Boivin, V., Siegmund, C., Inselmann, G., Lohse, M.J. and Boege, F. (1999) Autoantibodies activating human beta1-adrenergic receptors are associated with reduced cardiac function in chronic heart failure. Circulation, 99, 649-654. doi:10.1161/01.CIR.99.5.649

[8] Christ, T., Wettwer, E., Dobrev, D., Adolph, E., Knaut, M., Wallukat, G. and Ravens, U. (2001) Autoantibodies against the betal adrenoreceptor from patients with dilated cardiomyopathy prolong action potential duration and enhance contractility in isolated cardiomyocytes. Journal of Molecular and Cellular Cardiology, 33, 15151525. doi:10.1006/jmcc.2001.1414

[9] Mobini, R., Staudt, A., Felix, S.B., Baumann, G., Wallukat, G., Deinum, J., Svensson, H., Hjalmarson, A. and Fu, M. (2003) Hemodynamic improvement and removal of autoantibodies against beta 1 -adrenergic receptor by immunoadsorption therapy in dilated cardiomyopathy. Journal of Autoimmunity, 20, 345-350. doi:10.1016/S0896-8411(03)00042-8

[10] Belch, J.J., Bridges, A.B., Scott, N. and Chopra, M. (1991) Oxygen free radicals and congestive heart failure. British Heart Journal, 65, 245-248. doi:10.1136/hrt.65.5.245

[11] McCord, J.M. (1985) Oxygen-derived free radicals in postischemic tissue injury. The New England Journal of Medicine, 312, 159-163. doi:10.1056/NEJM198501173120305

[12] Tsutamoto, T., Wada, A., Matsumoto, T., Maeda, K., Mabuchi, N., Hayashi, M., Tsutsui, T., Ohnishi, M., Sawaki, M., Fujii, M., Matsumoto, T., Yamamoto, T., Horie, H., Sugimoto, Y. and Kinoshita, M. (2001) Relationship between tumor necrosis factor-alpha production and oxidative stress in the failing hearts of patients with dilated cardiomyopathy. Journal of the American College of Cardiology, 37, 2086-2092.

doi:10.1016/S0735-1097(01)01299-2

[13] Schimke, I., Müller, J., Priem, F., Kruse, I., Schön, B., Stein, J., Kunze, R., Wallukat, G. and Hetzer, R. (2001) Decreased oxidative stress in patients with idiopathic dilated cardiomyopathy one year after immunoglobulin adsorption. Journal of the American College of Cardiology, 38, 178-183. doi:10.1016/S0735-1097(01)01309-2
[14] Ikeda, U., Kasai, H., Izawa, A., Koyama, J., Yazaki, Y., Higuchi, M., Chang-Sung, Koh. and Yamamoto, K. (2008) Immunoadsorption therapy for patients with dilated cardiomyopathy and heart failure. Current Cardiology Reviews, 4, 219-222. doi:10.2174/157340308785160534

[15] Aso, S., Yazaki, Y., Kasai, H., Takahashi, M., Yoshio, T., Yamamoto, K. and Ikeda, U. (2009) Anti-beta1-adrenoreceptor autoantibodies and myocardial sympathetic nerve activity in chronic heart failure. International Journal of Cardiology, 131, 240-245. doi:10.1016/j.ijcard.2007.10.029

[16] Cavalleri, A., Colombo, C., Venturelli, E., Micelli, R., Mariani, L., Cornelli, U., Pala, V., Berrino, F. and Secreto, G. (2004) Evaluation of reactive oxygen metabolites in frozen serum samples. Effect of storage and repeated thawing. The International Journal of Biological Markers, 19, 250-253.

[17] Kakita, H., Hussein, M.H., Daoud, G.A., Kato, T., Murai, H., Sugiura, T., Mizuno, K., Yamada, Y., Ito, T., Fukuda, S., Kato, I., Suzuki, S. and Togari, H. (2006) Total hydroperoxide and biological antioxidant potentials in a neonatal sepsis model. Pediatric Research, 60, 675-679. doi:10.1203/01.pdr.0000245911.79943.8a

[18] Wallukat, G., Reinke, P., Dörffel, W.V., Luther, H.P., Bestvater, K., Felix, S.B. and Baumann, G. (1996) Removal of autoantibodies in dilated cardiomyopathy by immunoadsorption. International Journal of Cardiology, 54, 191-195. doi:10.1016/0167-5273(96)02598-3

[19] Staudt, A., Boehm, M., Knebel, F., Grosse, Y., Bischoff, C., Hummel, A., Dahm, J.B., Borges, A., Jochmann, N., Wernecke, K.D., Wallukat, G., Baumann, G. and Felix, S.B. (2002) Potential role of autoantibodies belonging to the immunoglobulin G-3 subclass in cardiac dysfunction among patients with dilated cardiomyopathy. Circulation, 106, 2448-2453.

doi:10.1161/01.CIR.0000036746.49449.64

[20] Siwik, D.A., Tzortzis, J.D., Pimental, D.R., Chang, D.L., Pagano, P.J., Singh, K., Sawyer, D.B. and Colucci, W.S. (1999) Inhibition of copperzinc superoxide dismutase induces cell growth, hypertrophic phenotype, and apoptosis in neonatal rat cardiac myocytes in vitro. Circulation Research, 85, 147-153. doi:10.1161/01.RES.85.2.147

[21] Giulivi, C., Boveris, A. and Cadenas, E. (1995) Hydroxyl radical generation during mitochondrial electron transfer and the formation of 8-hydroxydesoxyguanosine in mitochondrial DNA. Archives of Biochemistry and Biophysics, 316, 909-916.

[22] Siwik, D.A., Pagano, P.J. and Colucci, W.S. (2001) Oxidative stress regulates collagen synthesis and matrix metalloproteinase activity in cardiac fibroblasts. American Journal of Physiology - Cell Physiology, 280, C53-C60.

[23] Creemers, E.E., Cleutjens, J.P., Smits, J.F., Daemen and M.J. (2001) Matrix metalloproteinase inhibition after myocardial infarction: A new approach to prevent heart failure? Circulation Research, 89, 201-210. doi: $10.1161 / \mathrm{hh} 1501.094396$

[24] Torre-Amione, G. (2005) Immune activation in chronic heart failure. American Journal of Cardiology, 95, 3-8. doi:10.1016/j.amjcard.2005.03.006 
[25] Oda, S., Hirasawa, H., Shiga, H., Makanishi, K., Matsuda, K., Nakamura, M., Ikeda, H. and Sakai, M. (2004) Cytokine adsorptive property of various adsorbents in immunoadsorption columns and a newly developed adsorbent: an in vitro study. Blood Purification, 22, 530-536. doi: $10.1159 / 000082526$

[26] Müller, J., Wallukat, G., Dandel, M., Bieda, H., Brandes, K., Spiegelsberger, S., Nissen, E., Kunze, R. and Hetzer,
R. (2000) Immunoglobulin adsorption in patients with idiopathic dilated cardiomyopathy. Circulation, 101, 385391. doi:10.1161/01.CIR.101.4.385

[27] Dörffel, W.V., Wallukat, G., Dörffel, Y., Felix, S.B. and Baumann, G. (2004) Immunoadsorption in idiopathic dilated cardiomyopathy, a 3-year follow-up. International Journal of Cardiology, 97, 529-534. doi:10.1016/j.ijcard.2004.03.001 